\title{
MALDI Mass Spectrometric Analysis of Nonderivatized Steroids Using Cyclodextrin-supported 2,5-Dihydroxybenzoic Acid as Matrix
}

\author{
Jeongjin Son and Sangwon Cha* \\ Department of Chemistry, Hankuk University of Foreign Studies, Yongin, Kyunggi-Do 449-791, Korea \\ *E-mail: swcha@hufs.ac.kr \\ Received December 4, 2013, Accepted January 20, 2014
}

\begin{abstract}
Sex hormones are important metabolites in vertebrates' development and reproduction. For rapid screening sex hormones, matrix-assisted laser desorption/ionization (MALDI) mass spectrometry (MS) is one of the promising analytical platforms, but MALDI MS faces many challenges in detecting steroids such as low ionization efficiency and matrix background interference. One potential strategy to overcome matrix interference in the low $\mathrm{m} / \mathrm{z}$ region is using a cyclodextrin (CD)-supported matrix for steroid analysis since CDsupported matrixes are known to effectively suppress matrix-related ion signals. In this study, we aimed to find the optimal CD-supported matrix for the analysis of the nonderivatized sex steroids. Our results showed that the $\alpha \mathrm{CD}$-supported 2,5-dihydroxybenzoic acid (DHB) matrix efficiently ionized all three major classes of sex hormones, estrogens, androgens, and progestagens, with low or no matrix background and also with high sensitivity. In addition, the $\alpha \mathrm{CD}$-supported DHB matrix mainly generated molecular ions or protonated ions of sex hormones, and this enabled us to obtain information-rich tandem mass spectra which potentially lead to unambiguous identification of steroid species from complex metabolite mixtures.
\end{abstract}

Key Words : MALDI MS, Steroids, Estrogen, Estrone, Cyclodextrin

\section{Introduction}

Sex hormones are essential biomolecules in vertebrates and mainly involved in the maintenance of pregnancy, the development of secondary sex characteristics, and many diseases such as osteoporosis and breast cancer. There are three major classes of sex steroids, estrogens, androgens, and progestagens which have 18, 19, and 21 carbon atoms in their core structures, respectively.

In the analysis of sex hormones, matrix-assisted laser desorption/ionization (MALDI) mass spectrometry (MS) is a very attractive method because of its ability to analyze complex mixtures with a simple sample preparation protocol and also because of its potential as a high throughput screening platform. However, MALDI MS of neutral steroids is challenging with following reasons. First, ion yields of steroids in MALDI MS are usually very low since neutral steroids lack acidic or basic groups in their structures and this leads to poor sensitivity. Second, intense MALDI matrix ion signals in the low $\mathrm{m} / \mathrm{z}$ region often interfere with or suppress steroid signals.

In order to overcome these issues, many research efforts have been made so far. First, steroids can be derivatized to improve ion yields of them. For example, 3-oxo-sterols were derivatized with Girad P hydrazine to give charged Girad P hydrazones, and these derivatized steroids were easily detected as molecular ions $\left([\mathrm{M}]^{+}\right)$by conventional MALDI MS. ${ }^{1}$ In addition, these derivatized oxysterols also generated informationrich high energy collision induced dissociation (CID) tandem mass spectra.

Second, a high molecular weight matrix can be used to avoid the overwhelmed matrix ion signals in the low $\mathrm{m} / \mathrm{z}$ region. For example, a meso-tetrakis(pentafluorophenyl)porphyrin (F20TPP, MW 974.57) matrix was used for the analysis of the five neutral steroids from human urine. ${ }^{2}$ Third, a surface-assisted laser desorption/ionization (SALDI) MS platform or an alternative matrix such as nanomaterials can be employed to both improve ion yield and avoid matrix interference. For example, an activated carbon surface and $\mathrm{C}_{70}$ fullerene was used for the analysis of the nonderivatized steroids. ${ }^{3,4}$ Interestingly, with a carbon-based matrix, estrogens were more effectively detected in the negative ion mode while testosterone and progesterone were preferentially ionized in the positive ion mode. ${ }^{3}$ Gold nanoparticles were also used as a matrix for the detection of testosterone and progesterone. ${ }^{5}$

A cyclodextrin (CD)-supported matrix, originally developed by Korenaga and co-workers, is another effective way to reduce matrix interference. ${ }^{6-8} \mathrm{~A} C D$-supported matrix is a complex of CD and an organic MALDI matrix prepared by simple heated sonication, and their complex formation was confirmed by UV-VIS and ${ }^{1} \mathrm{H}$ NMR spectroscopy. ${ }^{67}$ With a $\mathrm{CD}$-supported matrix, matrix-related ions were significantly suppressed and therefore much cleaner mass spectra could be obtained for various small molecules from forensic samples. ${ }^{8}$

In present study, we developed a MALDI MS method for the analysis of the nonderivatized sex steroids by optimizing a CD-supported matrix system. We found that all major classes of sex hormones (Figure 1) were detected by MALDI with a clean background when using the $\alpha$ CD-supported 2,5dihydroxybenzoic acid (DHB) matrix. In addition, we also 

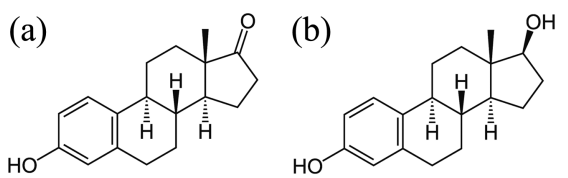

(c)

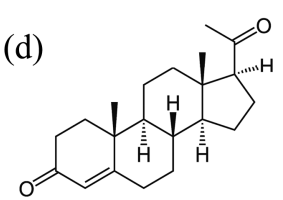

Figure 1. Chemical structures of sex hormones analyzed in this study: (a) estrone, (b) $\beta$-estradiol, (c) testosterone, and (d) progesterone.

confirmed that the $\alpha \mathrm{CD}$-supported DHB is a suitable matrix for producing information-rich product ion spectra of neutral steroids.

\section{Experimental}

Materials. Estrone (MW 270.37), $\beta$-estradiol (MW 272.38), testosterone (MW 288.42), progesterone (MW 314.46), trifluoroacetic acid (TFA, 99\% purity), 2,4,6-trihydroxyacetophenone (THAP), and cyclodextrins ( $\alpha-, \beta-$, and $\gamma$ - forms) were obtained from Sigma-Aldrich (St. Louise, MO, USA). A recrystallized DHB was purchased from Protea Biosciences (Morgantown, WV, USA). Aqueous solution of citratecapped gold nanoparticles $(0.05 \mathrm{mg} / \mathrm{mL}, 12.0 \pm 1.3 \mathrm{~nm}$ in diameter) was purchased from CNVision Co. (Seoul, Korea). All solvents except deionized water were obtained from Fisher Scientific (Fairlawn, NJ, USA).

Sample Preparation for MALDI MS Analysis. The standard solutions of steroids were prepared in ethanol with the concentration of $1.0 \mathrm{mg} / \mathrm{mL}$. The $\alpha \mathrm{CD}$-supported DHB matrix solution was prepared based on a previous protocol ${ }^{8}$ with some modification. Briefly, $10 \mathrm{mM} \alpha \mathrm{CD}$ solution in water and DHB solution $(20 \mathrm{mg} / \mathrm{mL})$ in $50 / 50$ acetonitrile/ water were prepared separately. Then, $20 \mu \mathrm{L}$ of DHB solution was mixed with $120 \mu \mathrm{L}$ of $\alpha \mathrm{CD}$ solution, and the mixture was sonicated for $10 \mathrm{~min}$ at $50{ }^{\circ} \mathrm{C}$. In case of the gold nanoparticle matrix, the solution obtained from CNVision Co. was directly used without further treatment.

For MALDI MS analysis, a steroid sample solution $(0.5$ $\mu \mathrm{L}$ ) was first spotted onto a 384-well MALDI target plate (ASTA Inc., Suwon, Korea), followed by $0.5 \mu \mathrm{L}$ matrix solution. After spotting, spotted samples were dried under a moderate vacuum $(\sim 50$ Torr $)$ at room temperature.

MALDI Mass Spectrometry. ABI 4800 Plus MALDITOF/TOF analyzer (Applied Biosystems, Foster City, CA) controlled by the 4000 Series Explorer ${ }^{\mathrm{TM}}$ software was used for MALDI MS and MS/MS analyses of steroids. The mass spectrometer was operated in the positive reflectron mode with a $20 \mathrm{kV}$ acceleration voltage. For a given sample spot, sub-spectra were collected from 20 different locations in the spot and then averaged to generate a final mass spectrum (800 laser shots in total per sample spot). In case of MS/MS experiments, high energy CID was performed using air as the collision gas at $1 \mathrm{kV}$ of collision energy. A DataExplorer

V4.8 (Applied Biosystems) and a mMass software (http:// www.mmass.org) ${ }^{9}$ were used for further processing mass spectra.

\section{Results and Discussion}

Optimization of a CD-Supported Matrix for Detection of Nonderivatized Steroids. We initially tested the previously reported $\alpha \mathrm{CD}$-supported THAP matrix for the steroid analysis. $^{7}$

Among steroids investigated, testosterone and proge-
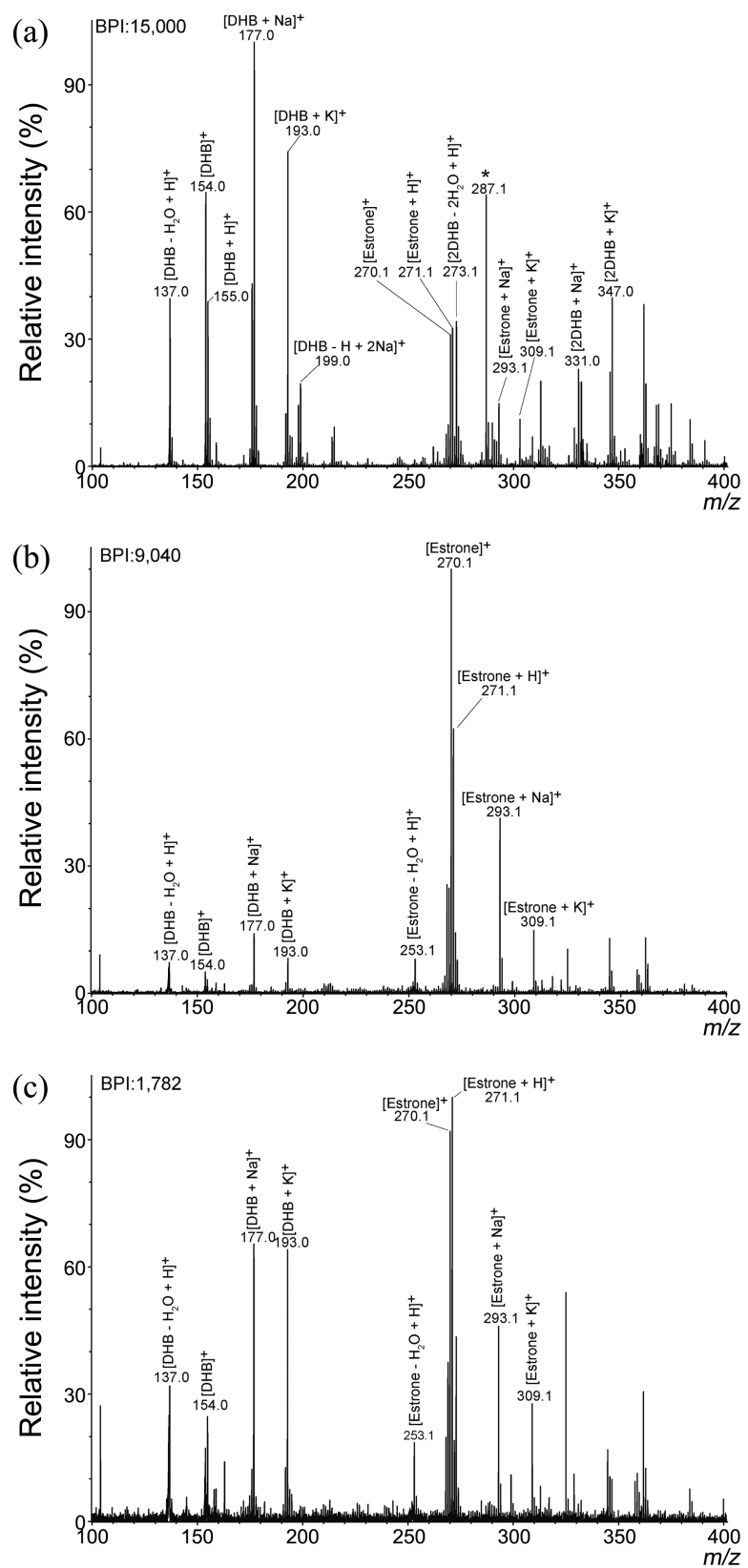

Figure 2. MALDI mass spectra of estrone (a) with the DHB-only matrix, (b) with the $\alpha \mathrm{CD}$-supported DHB matrix prepared by heated sonication for $10 \mathrm{~min}$, and (c) with the DHB plus $\alpha \mathrm{CD}$ matrix prepared by 5 min vortexing. The peak at $m / z 287$ in (a) is an unknown peak coming from either a DHB matrix or a MALDI target plate. BPI stands for absolute base peak intensity. 
sterone were efficiently ionized by the $\alpha \mathrm{CD}$-supported THAP matrix with minimal matrix interference similar to the previous study. ${ }^{8}$

However, 18-carbon sex hormones, estrogens (estrone and $\beta$-estradiol) were not ionized by the $\alpha \mathrm{CD}$-supported THAP matrix or by the THAP-only matrix. Therefore, we tested other organic MALDI matrixes in order to find a suitable matrix for ionization of sex hormones.

Among matrixes tested, DHB showed an acceptable performance in detecting estrogens as shown in Figure 2(a). With a DHB-only matrix, estrone was detected as $\mathrm{M}^{+}$at $\mathrm{m} / \mathrm{z}$ $270,[\mathrm{M}+\mathrm{H}]^{+}$at $m / z 271,[\mathrm{M}+\mathrm{Na}]^{+}$at $m / z 293$, and $[\mathrm{M}+$ $\mathrm{K}^{+}$at $m / z 309$, but the mass spectrum was dominated by ion signals originated from a DHB matrix. In order to achieve suppression of matrix-related signals, various sizes of CDs $(\alpha-, \beta-$, and $\gamma$ - forms) were incorporated into the DHB matrix and tested.

Among CD-DHB complexes investigated, only $\alpha \mathrm{CD}-\mathrm{DHB}$ showed a matrix suppression effect with detectible steroid ion signals, and therefore further optimization in matrix preparation was carried out with $\alpha \mathrm{CD}$ only. In the previous study, the optimized molar ratio of THAP and $\alpha \mathrm{CD}$ was $1: 1$ and the concentration was $\sim 8.8 \mathrm{mM}$ each in the final matrix solution. ${ }^{6,7}$ However, the $\alpha \mathrm{CD}$-supported DHB matrix prepared from the equimolar mixture of $\mathrm{DHB}$ and $\alpha \mathrm{CD}(\sim 8.8$ $\mathrm{mM}$, respectively) did not ionize steroids efficiently although this matrix showed good matrix suppression. Therefore, we generated matrix solutions with various molar ratios of DHB and $\alpha \mathrm{CD}$ ranging from 10:1 to $1: 100$ (DHB: $\alpha \mathrm{CD}$ ), and tested these solutions against steroids. From this investigation, we found that a molar ratio of near 2:1 (DHB: $\alpha \mathrm{CD}$, $18.5 \mathrm{mM}: 8.6 \mathrm{mM}$ ) produced the best result. Further investigation is needed to clearly understand complex formation or host-guest interaction between DHB and $\alpha \mathrm{CD}$.

Figure 2(b) shows the MALDI mass spectrum of estrone with the optimized $\alpha \mathrm{CD}$-supported DHB matrix. Compared to Figure 2(a), ion signals from DHB were clearly suppressed while estrone ion signals were enhanced. It should be also noted that the $\alpha \mathrm{CD}$-supported DHB matrix generated mainly molecular ions or protonated ions which is preferred in structural analysis of steroids by MS/MS. This will be discussed in more detail in the next chapter.

In addition, we examined whether heated sonication is also required for the preparation of the $\alpha \mathrm{CD}$-supported DHB matrix, and we confirmed that the $\alpha \mathrm{CD}$-supported $\mathrm{DHB}$ matrix prepared by heated sonication gave the better suppression of matrix ion signals as well as the more enhancement of steroid signals than one prepared by the simple vigorous mixing as shown in Figure 2(b) and 2(c). At least 5 min sonication at $50^{\circ} \mathrm{C}$ was required for the effective formation of $\alpha \mathrm{CD}-\mathrm{DHB}$ complex.

Figure 3 shows MALDI mass spectra of other sex steroids with the optimized $\alpha \mathrm{CD}$-supported DHB matrix. All mass spectra showed molecular or protonated ions of steroids as a major species with low or no matrix-related ion signals. The limits of detection $(\mathrm{S} / \mathrm{N}>5)$ of estrone and $\beta$-estradiol were 50 pmol per sample spot and the limits of detection (S/
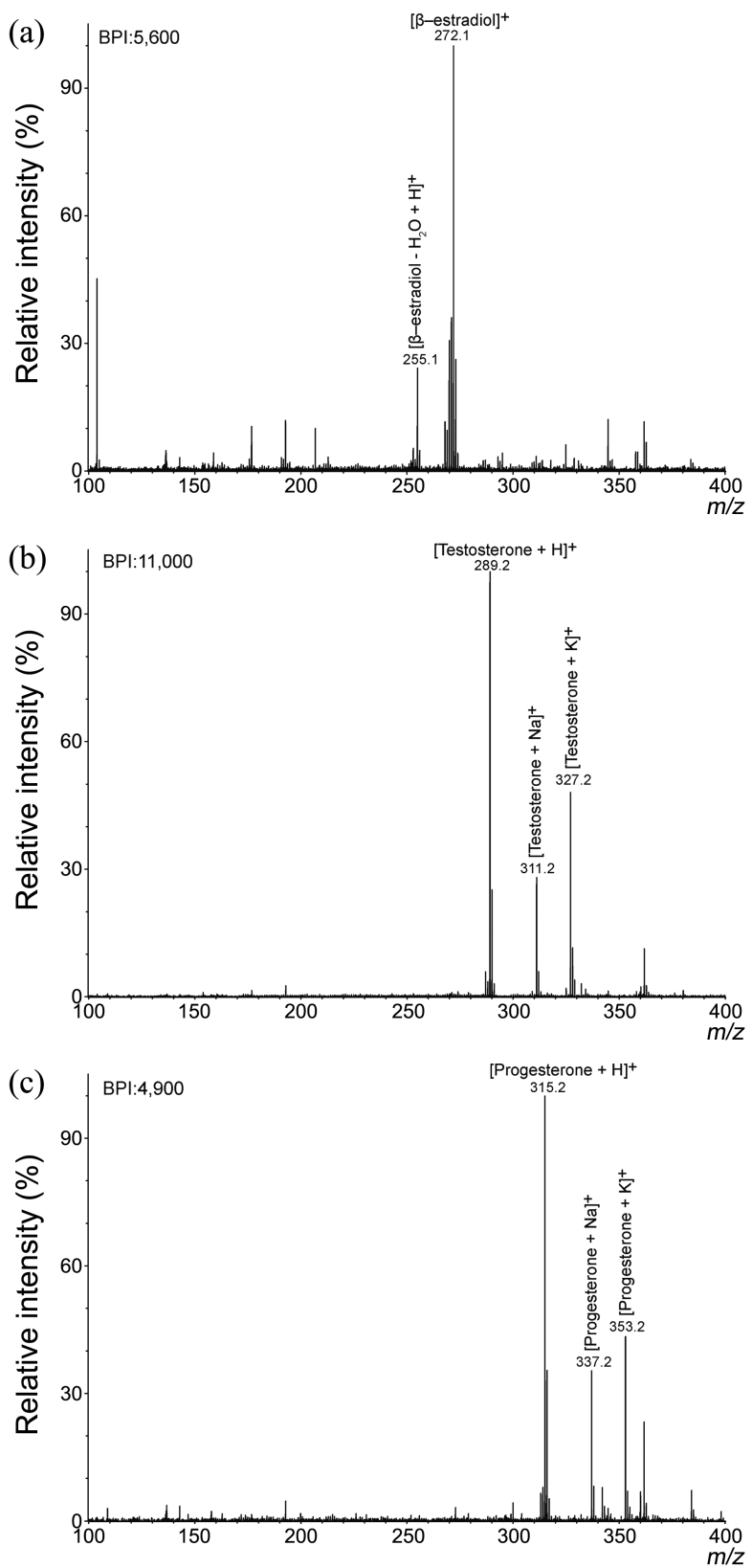

Figure 3. MALDI mass spectra of nonderivatized sex hormones with the $\alpha \mathrm{CD}$-supported DHB matrix: (a) $\beta$-estradiol, (b) testosterone, and (c) progesterone. BPI stands for absolute base peak intensity.

$\mathrm{N}>5$ ) of progesterone and testosterone were 2 pmol per sample spot. These results were comparable or better than those obtained by carbon material- or gold nanoparticlebased LDI MS methods. ${ }^{3-5}$

Structural Analysis of Nonderivatized Steroids by $\alpha$ CDsupported Matrix-based MALDI MS/MS. In previous reports, laser desorption/ionization (LDI) MS methods using an activated carbon surface or gold nanoparticle as a matrix mainly generated sodium adduct ions of sex steroids in the positive ion mode. ${ }^{4,5}$ This alkali cationization of steroids is not a problem when it is possible to identify steroids only by their $m / z$ values. However, in case of analyzing a complex steroid mixture, identification of steroid species only by their 

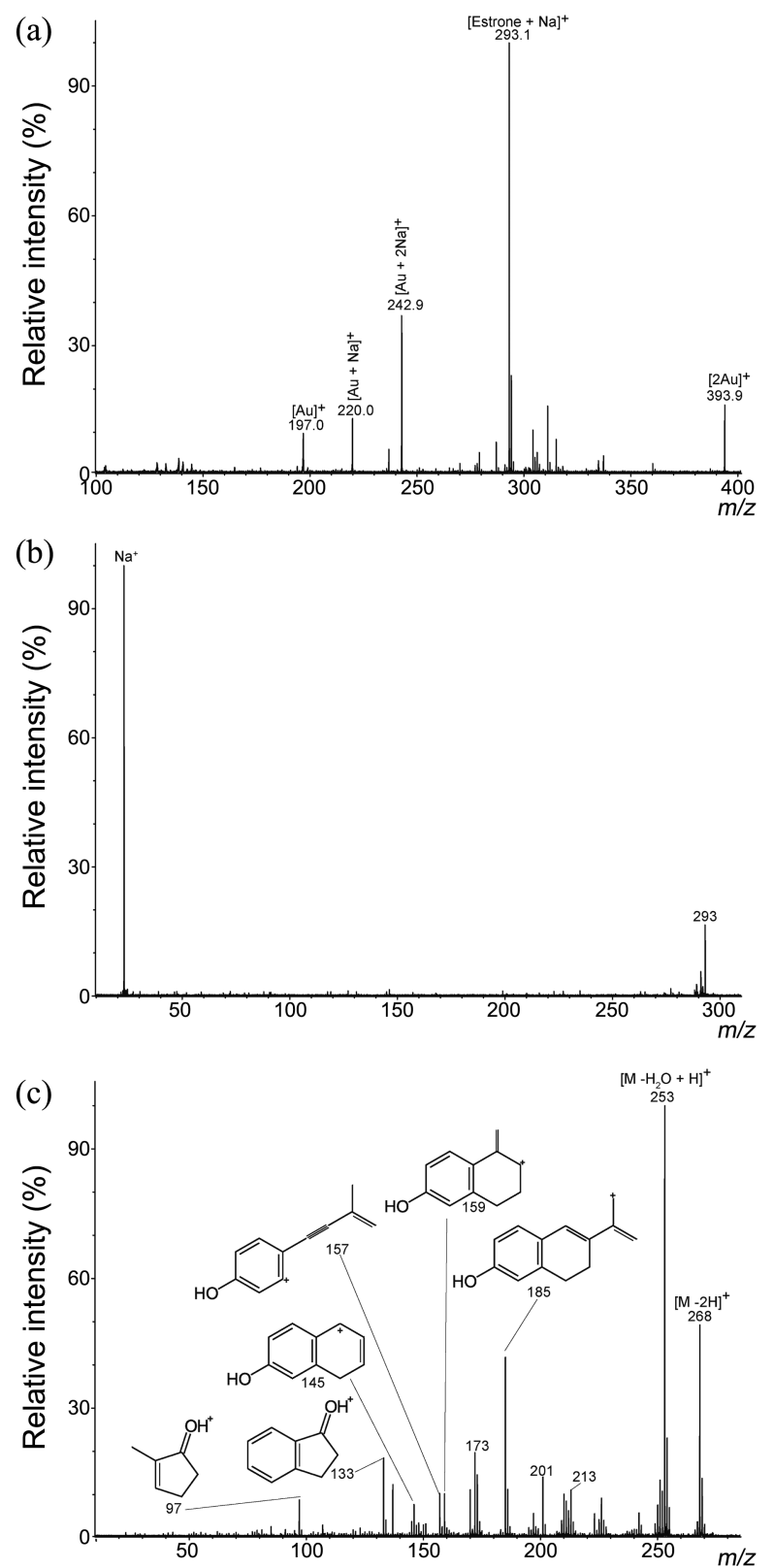

Figure 4. (a) LDI mass spectrum of estrone with an aqueous solution of gold nanoparticles as a matrix. (b) MALDI TOF/TOF mass spectrum of [estrone $+\mathrm{Na}]^{+}$ion detected at $m / z 293$ in (a). (c) MALDI TOF/TOF mass spectrum of [estrone $]^{+}$and [estrone $\left.+\mathrm{H}\right]^{+}$ ions detected at $m / z 270$ and 271 in the MALDI mass spectrum of estrone with the $\alpha \mathrm{CD}$-supported DHB matrix (Figure 2(b)). Chemical structures in the spectrum are possible fragment ions from [estrone $+\mathrm{H}]^{+}$based on previous studies. ${ }^{1,10-12}$

$\mathrm{m} / \mathrm{z}$ values is not feasible since many isobaric steroids or other small metabolites can be present in a mixture. Therefore, structural analysis by MS/MS is usually required and performed. In such case, alkali cationized ions are potentially problematic because these ions do not often provide information-rich $\mathrm{MS} / \mathrm{MS}$ due to their less covalent nature in ion formation.

Figure 4(a) shows the MALDI mass spectrum of estrone with an aqueous solution of gold nanoparticles as a matrix.
Similar to the previous report, ${ }^{5}$ estrone was exclusively and easily detected as a $[\mathrm{M}+\mathrm{Na}]^{+}$form. In MS/MS of this ion, however, almost no fragment ion except sodium ion was observed (Figure 4(b)). In contrast, the product ion spectrum of the molecular ion and the protonated ion appeared in the MALDI mass spectrum of estrone with the $\alpha C D$-supported DHB matrix (Figure 2(b)) provided detailed structural information as illustrated in Figure 4(c). Chemical structures presented in Figure 4(c) are possible product ions of protonated estrone ions. ${ }^{1,10-12}$ This suggests that $\alpha C D$-supported DHB MALDI MS has a potential advantage over other SALDI MS methods in structural analysis of neutral steroids and also in complex steroid mixture analysis.

\section{Conclusion}

In this study, we optimized a CD-supported matrix for the detection of the nonderivatized steroids by MALDI MS. By using the $\alpha \mathrm{CD}$-supported DHB matrix, all three major classes of sex steroids were detected with high sensitivity while background ions originated from a DHB matrix were effectively suppressed. In addition, since major ion forms of steroids produced by the $\alpha \mathrm{CD}$-supported DHB matrix were molecular or protonated ions, informative fragmentation patterns could be obtained by high energy CID MS/MS which is a potential benefit in complex steroid mixture analysis. Future studies will include the application of the method to the steroid analysis of complex biological fluids or tissues, the investigation of the $\alpha \mathrm{CD}-\mathrm{DHB}$ interaction properties, and the further optimization of the method for improving sensitivity.

Acknowledgments. This work was supported by Hankuk University of Foreign Studies Research Fund of 2013.

\section{References}

1. Wang, Y. Q.; Hornshaw, M.; Alvelius, G.; Bodin, K.; Liu, S. Y.; Sjovall, J.; Griffiths, W. J. Anal. Chem. 2006, 78, 164.

2. Kosanam, H.; Prakash, P. K.; Yates, C. R.; Miller, D. D.; Ramagiri, S. Anal. Chem. 2007, 79, 6020.

3. Montsko, G.; Vaczy, A.; Maasz, G.; Mernyak, E.; Frank, E.; Bay, C.; Kadar, Z.; Ohmacht, R.; Wolfling, J.; Mark, L. Anal. Bioanal. Chem. 2009, 395, 869.

4. Guild, G. E.; Lenehan, C. E.; Walker, G. S. Int. J. Mass Spectrom. 2010, 294, 16.

5. Wu, H.-P.; Yu, C.-J.; Lin, C.-Y.; Lin, Y.-H.; Tseng, W.-L. J. Am. Soc. Mass Spectrom. 2009, 20, 875.

6. Yamaguchi, S.; Fujita, T.; Fujino, T.; Korenaga, T. Analyt. Sci. 2008, 24, 1497.

7. Fujita, T.; Fujino, T.; Hirabayashi, K.; Korenaga, T. Analyt. Sci. 2010, 26, 743 .

8. Yonezawa, T.; Asano, T.; Fujino, T.; Nishihara, H. Chem. Phys. 2013, 419, 17

9. Niedermeyer, T. H. J.; Strohalm, M. Plos One 2012, 7.

10. Griffiths, W. J.; Wang, Y.; Alvelius, G.; Liu, S.; Bodin, K.; Sjovall, J. J. Am. Soc. Mass. Spectrom. 2006, 17, 341.

11. Biancotto, G.; Angeletti, R.; Traldi, P.; Silvestri, M.; Saccon, M.; Guidugli, F. J. Mass. Spectrom. 2002, 37, 1266.

12. Bourcier, S.; Poisson, C.; Souissi, Y.; Kinani, S.; Bouchonnet, S.; Sablier, M. Rapid Commun. Mass Spectrom. 2010, 24, 2999. 